\title{
Prevalence of mental disorders in a prison population in Durban, South Africa
}

\author{
S Naidoo, DL Mkize
}

Department of Psychiatry, Nelson R Mandela School of Medicine, University of KwaZulu-Natal, KwaZulu-Natal, South Africa

\begin{abstract}
Objective: The aim of this study was to determine the prevalence of serious mental disorders in a prison population in Durban, South Africa, one of the largest prisons in the Southern hemisphere. Method: 193 prisoners were interviewed using the Mini Neuro-psychiatric Interview, a screening questionnaire and a demographic questionnaire. Results: The study demonstrated that 55.4\% of prisoners had an Axis 1 disorder. The commonest disorder being substance and alcohol use disorders ( 42.0\%). 23.3\% of prisoners were diagnosed with current psychotic, bipolar, depressive and anxiety disorders. 46.1\% were diagnosed with antisocial personality disorder. The majority of prisoners diagnosed as having an Axis l disorder in this study, were neither diagnosed nor treated in prison. Conclusion: There is a high prevalence of mental disorders among prisoners in a prison population in Durban, South Africa. The majority of these prisoners are untreated in prison, related to non detection of the mental disorder. Greater mental health awareness and provision of mental health services focusing on staff training programmes to detect mental illnesses are needed and further research is recommended throughout South Africa.
\end{abstract}

Keywords: Prevalence; Mental disorders; Prison population; South Africa

Received: $23-11-2010$

Accepted: $04-04-2011$

doi: http://dx.doi.org/10.4314/ajpsy.v15il.4

\section{Introduction}

South Africa has a rapidly growing prison population and has the most prisoners amongst all African countries. ${ }^{1}$ It also has the world's seventh highest number of prisoners, outranking countries with up to nearly five times its population. In the continent of Africa, South Africa has the highest ratio of prisoners to total population; 348 prisoners for every 100, 000 people. ${ }^{1}$

International studies have consistently shown a high prevalence of mental disorders among prisoners. ${ }^{2-5}$ National studies conducted in New Zealand on all female prisoners and remanded male prisoners as well as a cohort of 18 percent of sentenced male prisoners from every prison, showed markedly elevated prevalence rates for major mental disorders in prisoners compared to community samples. ${ }^{2}$ Common disorders included substance use disorders,

\section{Correspondence}

Dr S. Naidoo

Department of Psychiatry, Nelson R Mandela School of Medicine,

Durban, South Africa

email: drsnaidoo@hotmail.com psychotic disorders, major depressive disorder, bipolar mood disorder, obsessive compulsive disorder and post traumatic stress disorder. They also found that there was a high rate of co-morbidity with substance use disorders amongst those diagnosed with major Axis 1 disorders. Significantly the study also showed that a large proportion of those found to be depressed or psychotic were not receiving treatment.

Butler et al in New South Wales, Australia screened both sentenced and reception prisoners and found that $43 \%$ of those prisoners screened had at least one of the following mental diagnoses : psychosis, anxiety or affective disorders in the past 12 month.s. ${ }^{3}$

A study in Canada comparing the prevalence of mental disorders in prisoners and the community found a very high rate of Axis 1 disorders among prisoners. ${ }^{4}$ Prisoners had a 91,7\% lifetime prevalence of psychiatric disorders while $76,7 \%$ showed symptoms in the past six months. This study also found a high rate of comorbidity with substance use disorders.

Although rates of mental disorders among prison populations are well known in western countries, it remains unknown whether these findings are applicable to non- 
western countries. There are currently no available data in South Africa. It would thus be important to determine whether South African prison populations show similar psychiatric morbidity and whether in fact further research in this field is necessary. The aim of this study was to determine the prevalence of mental disorders amongst prisoners at a prison in Durban, one of the largest prisons in South Africa and in the Southern Hemisphere. It accommodates approximately eleven thousand prisoners.

It is hoped that the findings of this study will result in an awareness to the high prevalence of mental disorders amongst prisoners and that it will influence future decisionmaking and planning for mental healthcare services at this prison and other prisons in South Africa.

The term " prisoner" is no longer used in South African Correctional Services; however for the sake of comparison to international studies, it shall be used in this study as a collective term to describe both awaiting trial and sentenced prisoners. ${ }^{6}$ Awaiting trial prisoners are now referred to as inmates and sentenced prisoners as offenders.

\section{Methods \\ Ethical considerations}

Prisoners are traditionally viewed as a vulnerable group in terms of being used as research subjects. ${ }^{7}$ Thus stringent ethical considerations were rigorously applied. The study was approved by the KwaZulu Natal Biomedical Research Ethics Committee. Approval was also obtained from the Department of Correctional Services Head Office in Pretoria. Participants informed consent was also obtained. Prisoners are considered vulnerable because they are involuntarily institutionalized and are thus prone to coercion and undue influence. They have been especially vulnerable to invasive research in the past. Thus prisoners who were randomly selected were verbally counselled regarding their ability to choose whether or not they wanted to participate in the study and the fact that they would not be victimized should they choose not to participate. They were also informed that any information disclosed during the interview would remain confidential and they were assured of anonymity. They also received an information leaflet with all the relevant details of the study and they retained the right to withdraw from the study at any point in time. This research was also not physically invasive as it involved an interview with the principal investigator.

\section{Instruments}

The Mini International Neuro-psychiatric Interview (MINI) was used as the instrument to screen prisoners for the presence or absence of mental illness. The MINI was designed as a brief structured interview for the major Axis 1 psychiatric disorders for DSM IV and ICD 10. Validity and reliability studies done show that the MINI has acceptably high validation and reliability scores and in addition it can be administered in a relatively short period of time. ${ }^{8}$ The MINI was chosen because it comprised sections examining psychotic, mood, anxiety, substance use and eating disorders. It also screened for antisocial personality disorder. These sections were chosen as they identified the most common and clinically significant disorders in the prison setting.
The services of an interpreter were enlisted to improve the quality of responses from the participants. A Zulu translation manual of the MINI was obtained to guide the interpreter so that accurate responses were recorded. This was a crucial factor since the majority of participants spoke isiZulu as their first language.

\section{Design}

This was a cross-sectional point prevalence study carried out at Westville Correctional Centre during the period January to February 2009. The sampling frame was approximately 9734 prisoners.

A comprehensive list was obtained from all 4 sections of the prison Medium A consisted of 4248 awaiting trial prisoners, Medium B consisted of 4196 sentenced prisoners, Medium C consisted of 947 male prisoners who were prerelease serving the terminal part of their sentences and lastly, the female section consisting of 343 awaiting trial and sentenced prisoners. The youth centre consisting of approximately 800 juveniles below the age of 18 were excluded. Prisoners are separated according to security risk that is maximum, medium and minimum security.

\section{Sampling}

Stratified systematic random sampling was done to ensure all sectors were proportionally represented. It was determined statistically that 193 prisoners needed to be interviewed in order to power the study in achieving a 95\% confidence interval for evaluation of the results. A refusal rate of 30\% was anticipated and thus 250 participants were selected. A significant proportion of those selected either refused, had already been released or were attending court (in the case of awaiting trial prisoners). Thus the target sample of 193 subjects was eventually obtained of the 250 selected initially.

\section{Interview process}

The interview process comprised four sections: informed consent (which included an information leaflet provided to each participant), demographic questionnaire, screening questionnaire and administration of MINI. Participants were all counselled and only those deemed capable of giving informed consent were recruited into the study. Participants were assured of confidentiality and were also informed that the interview could in no way assist them with their court proceeding, parole, sentencing or appeal. Those who were found to have a mental disorder were offered the option of being referred to the Westville's correctional services psychiatric and psychological services for the appropriate intervention.

All 193 participants were interviewed by the principal investigator. Demographic data was obtained from official prison records. Of the 193 participants recruited, 8 were females and 185 were males, this representing the demographic ratio at Westville Correctional Centre. The group included both awaiting trial and sentenced prisoners.

\section{Statistical analysis}

Statistical analysis was done using SPSS (Statistical Package for the Social Sciences) version 15.0 (SPSS Inc., Chicago, Ill., USA). ${ }^{9}$ 
Frequency tables, bar and pie charts were used to describe categorical variables. Pearson's chi square test was used to compare current disorders between awaiting trial and sentenced prisoners. A p value $<0.05$ was considered as statistically significant.

\section{Results}

\section{Socio-demographic characteristics}

The majority were single, employed, from urban areas and had a secondary level of education (see Table I). Prisoners had a mean age of 30,5 years with a standard deviation of 9.0 years and a range from 18 to 69 years. The majority of prisoners were African (90,7\%). This over-representation of African prisoners reflects the demographics of the prisoner population at this prison.

\begin{tabular}{|c|c|c|c|}
\hline & & Count & Column N\% \\
\hline GENDER & $\begin{array}{l}\text { FEMALE } \\
\text { MALE }\end{array}$ & $\begin{array}{l}8 \\
185\end{array}$ & $\begin{array}{l}4.1 \% \\
95.9 \%\end{array}$ \\
\hline ETHNICITY & $\begin{array}{l}\text { AFRICAN } \\
\text { COLORED } \\
\text { INDIAN } \\
\text { WHITE } \\
\text { OTHER }\end{array}$ & $\begin{array}{l}175 \\
6 \\
10 \\
1 \\
1\end{array}$ & $\begin{array}{l}90.7 \% \\
3.1 \% \\
5.2 \% \\
.5 \% \\
.5 \%\end{array}$ \\
\hline MARITAL STATUS & $\begin{array}{l}\text { DIVORCE } \\
\text { MARRIED } \\
\text { SINGLE } \\
\text { WIDOWED }\end{array}$ & $\begin{array}{l}7 \\
17 \\
168 \\
1\end{array}$ & $\begin{array}{l}3.6 \% \\
8.8 \% \\
87.0 \% \\
.5 \%\end{array}$ \\
\hline EDUCATION & $\begin{array}{l}\text { ILLITERAT } \\
\text { PRIMARY } \\
\text { SECOND } \\
\text { TERTIARY }\end{array}$ & $\begin{array}{l}5 \\
41 \\
139 \\
8\end{array}$ & $\begin{array}{l}2.6 \% \\
21.2 \% \\
72.0 \% \\
4.1 \%\end{array}$ \\
\hline EMPLOYMENT & $\begin{array}{l}\text { EMPLOY } \\
\text { SELF-EMP } \\
\text { UNEMP }\end{array}$ & $\begin{array}{l}96 \\
15 \\
82\end{array}$ & $\begin{array}{l}49.7 \% \\
7.8 \% \\
42.5 \%\end{array}$ \\
\hline
\end{tabular}

\section{Criminological characteristics}

Crimes prisoners were charged with included violent crimes (eg murder, assault, armed robbery and kidnapping), nonviolent crimes (eg fraud, theft, and housebreaking), sex crimes (eg rape and indecent assault) and drug related offences (eg possession of or dealing in illegal substances).

Of the 193 prisoners, 120 were sentenced prisoners and 73 were awaiting trial. 26 of the 120 sentenced prisoners were repeat offenders giving a recidivism rate of $21,7 \%$.

\section{Prevalence rates}

A high prevalence (55.4\%) of Axis 1 disorders was found. Excluding substance and alcohol use disorders, 23, 3\% had current Axis 1 disorders of psychosis, bipolar, depressive and anxiety disorders (Figure 1).

The most common Axis 1 disorder diagnosed was substance (including alcohol) use disorders with a total of $42,0 \%$. This represents the prevalence rate for the past 12 month. The most common substance misused was cannabis (Figure 2). 13.16\%

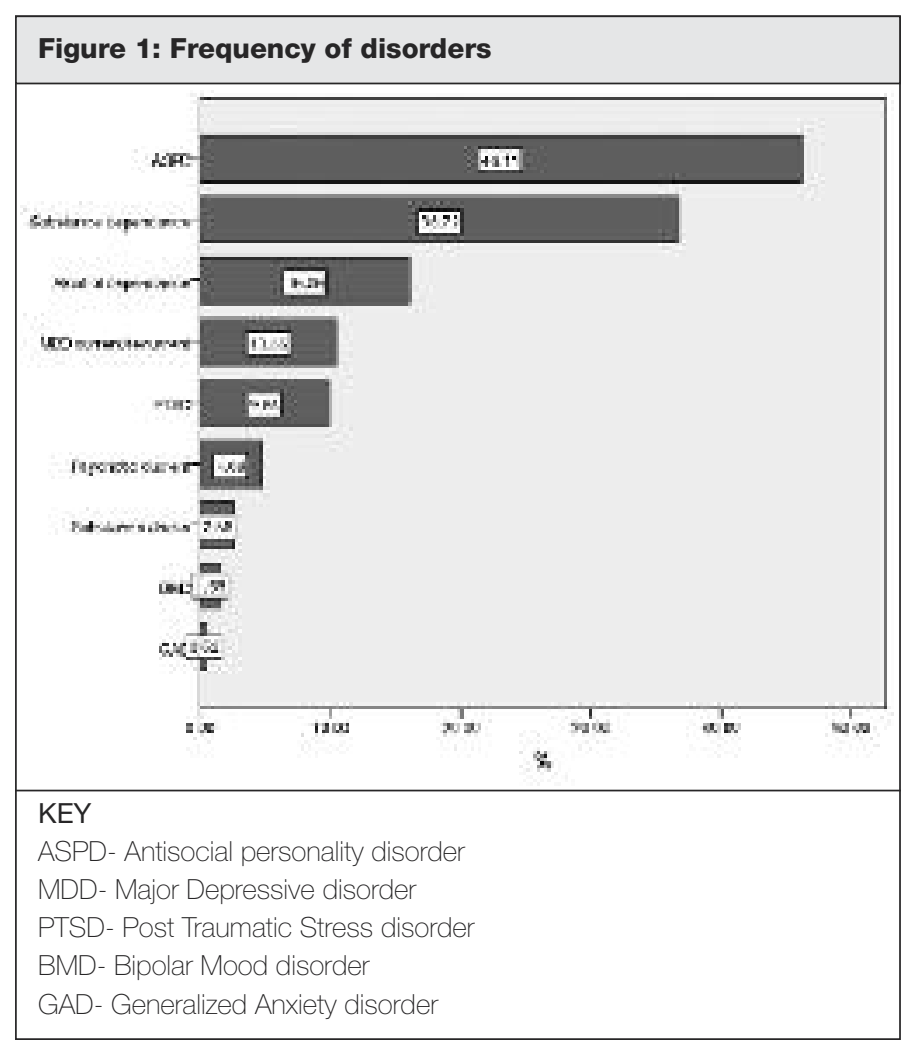

$(n=10)$ of prisoners abused more than one substance. The various combinations of substances are shown in Table II. There was a significant comorbidity between prisoners diagnosed with

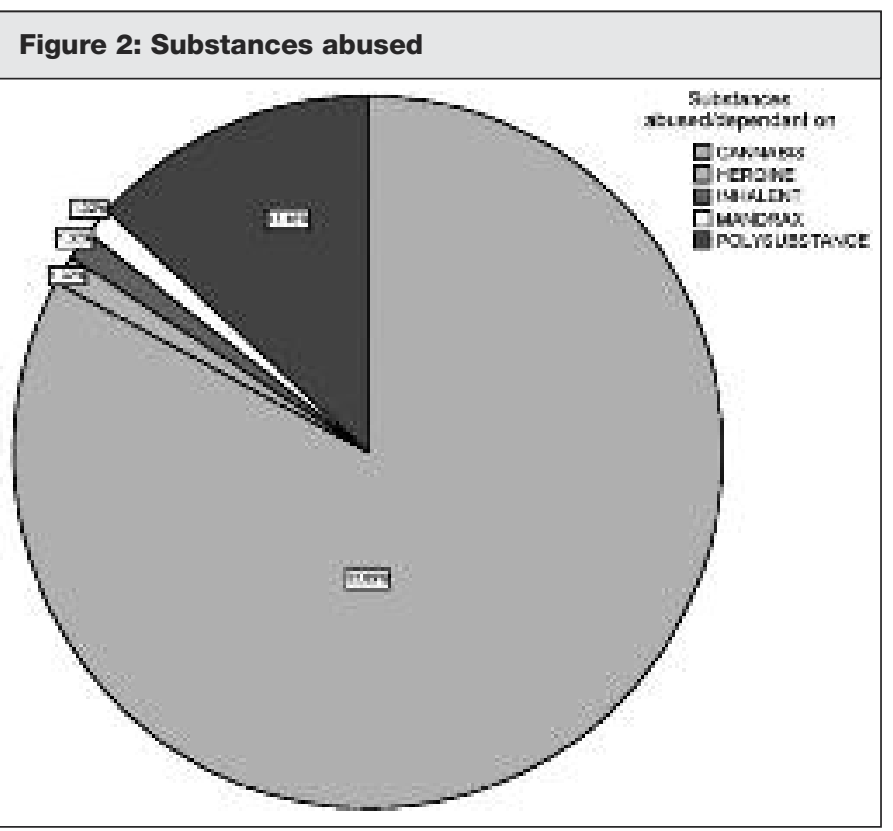

Table II: Polysubstance combinations

\begin{tabular}{|l|l|l|}
\hline & Frequency & Percent \\
\hline Cannabis and Coccaine & $n=2$ & 20 \\
Cannabis and Heroine & $n=3$ & 30 \\
Cannabis and Mandrax & $n=4$ & 40 \\
Cannabis, Heroine and Mandrax & $n=1$ & 10 \\
\hline Total & $n=10$ & 100 \\
\hline
\end{tabular}


current Axis 1 disorders and substance (including alcohol) use disorders (Table III).

\begin{tabular}{l} 
Table III: Comorbidity between current Axis 1 disorders and \\
SUD \\
\hline
\end{tabular}

Other common disorders included major depressive disorder and post traumatic stress disorder with lower rates of psychotic disorders, bipolar mood disorder and generalized anxiety disorder.

Currently $10.4 \%$ of prisoners were diagnosed with a major depressive disorder, while 14.5\% had experienced a major depressive episode in the past. The lifetime prevalence of depression was 24. 9\%.

Current psychotic disorders were diagnosed in 4.7\%, with a slightly higher lifetime prevalence of 7.3\%. The MINI however does not distinguish between schizophrenia, schizophreniform and brief psychotic disorder.

89 out of the 193 prisoners (46.1\%) were diagnosed with antisocial personality disorder (ASPD). A significant proportion of those diagnosed with current Axis 1 disorders had comrbid ASPD as shown in Table IV.

Table IV : Comorbidity between current Axis 1 disorders and
ASPD
\begin{tabular}{|l|l|l|l|}
\hline & Total & No. with comorbid ASPD & Percent \\
\hline Psychosis & $n=9$ & $n=5$ & 55,9 \\
MDD & $n=20$ & $n=6$ & 30,0 \\
BMD & $n=3$ & $n=2$ & 66,7 \\
PTSD & $n=19$ & $n=9$ & 47,3 \\
GAD & $n=1$ & $n=0$ & 0 \\
\hline
\end{tabular}

If one examines the two population groups of awaiting trial and sentenced prisoners separately (Table V), the following relationships are noted: Current psychotic disorders were more common among awaiting trial prisoners and current major depressive disorders as well as bipolar mood disorder were more common among sentenced prisoners, however the only statistically significant association was that awaiting trial prisoners were more likely to suffer from PTSD than sentenced prisoners. A study in Australia found that PTSD was the most common disorder diagnosed among prisoners. ${ }^{3}$

\section{Discussion}

\section{Prevalence rates}

This was the first ever study undertaken in South Africa to determine the prevalence rate of mental disorders amongst prisoners.

Overall, in keeping with international studies, high rates of mental disorders were detected amongst prisoners. 55.4\% of prisoners had a current Axis 1 disorder, this includes substance and alcohol use disorders. Significantly prisoners reported having access to cannabis while in prison; however they reported poor access to alcohol in prison. Substance abuse should be a major focus of rehabilitative intervention to prevent predisposition to mental illness as well as to ensure that prisoners are drug-free on release. Releasing prisoners who are still using substances back into the community will put them at higher risk of recidivism. ${ }^{10}$

However excluding substance and alcohol use disorders, only 23.3\% had current Axis 1 disorders i.e. psychotic bipolar, and depressive and anxiety disorders.

Although the reported prevalence of post traumatic stress disorder (PTSD) was 9, 8\%, many prisoners reported subsyndromal PTSD. Many had witnessed violent crimes for eg. murder of friends or family in their communities as well as having been witness to gangsterism and murder whilst in prison. They thus displayed some but not the full syndrome of PTSD symptomatology. This attenuated anxiety response could possibly be accounted for as a result of resilience or habituation since many of these prisoners were raised in communities where violence and crime was rife. Ironically the wider community perceives prisoners as traumatizers, but as we found in our study, they are also a traumatized group. ${ }^{5}$

A large proportion of prisoners (46.1\%) were diagnosed with antisocial personality disorder (ASPD). This is very significant as there is evidence that treatment of axis 1 disorders in people with ASPD is more complicated and therefore high rates of ASPD poses additional short and long term challenges for psychiatric services. ${ }^{11}$

A significant finding was that, according to prisoners self report, there was a huge disparity between the prevalence rates of mental disorders as diagnosed by the study and those who were detected and or being treated in prison.

8 out of 9 prisoners (i.e. almost 89\%) diagnosed as currently experiencing a psychotic disorder were not diagnosed or treated in prison.

All prisoners currently suffering from a major depressive disorder were not diagnosed or treated.

Two of the prisoners diagnosed as having BMD were

\section{Table V: Comparison of current disorders between awaiting trial and sentenced prisoners}

\begin{tabular}{|c|c|c|c|c|c|}
\hline & $\begin{array}{l}\text { Current psychotic } \\
\text { disorder }\end{array}$ & $\begin{array}{l}\text { Current major } \\
\text { depressive disorder }\end{array}$ & Bipolar mood disorder & Current PTSD & $\begin{array}{l}\text { Lifetime antisocial } \\
\text { personality disorder }\end{array}$ \\
\hline $\begin{array}{l}\text { Awaiting trial }(n=73) \\
\text { Sentenced prisoners }(n=120) \\
p \text { value }\end{array}$ & $\begin{array}{l}6,8 \%(n=5) \\
3,3 \%(n=4) \\
0.261\end{array}$ & $\begin{array}{l}8,2 \%(n=6) \\
11,7 \%(n=14) \\
0.446\end{array}$ & $\begin{array}{l}0 \%(n=0) \\
2,5 \%(n=3) \\
0.291\end{array}$ & $\begin{array}{l}17,8 \%(n=13) \\
5,0 \%(n=6) \\
0.004\end{array}$ & $\begin{array}{l}45.2 \%(n=33) \\
46.7 \%(n=56) \\
0.843\end{array}$ \\
\hline
\end{tabular}


never diagnosed nor treated. The third prisoner with bipolar mood disorder was previously diagnosed as having a mental disorder but was currently not on any prophylactic treatment despite having multiple previous mood episodes in the past.

All 19 prisoners diagnosed with PTSD by the study were not diagnosed nor treated.

Those participants classified as being suicidal (medium or high scores) had in the last month experienced suicidal ideation. The two prisoners with high suicidality scores were found to be at high risk whilst the two with medium scores were not actively suicidal. Only 4, 1\% of prisoners interviewed had ever made a past suicide attempt. Bland et al in Canada revealed that $22,8 \%$ of prisoners had at some point made a suicide attempt. 4 The low rate of suicidality amongst South African prisoners could partly be due to the fact that the majority of the prisoners were African Black males as opposed to the study by Bland et al in which the majority of prisoners were Caucasian. Suicide rates have been noted to be higher amongst Caucasians than Blacks in South Africa. ${ }^{12}$

Participants past and current medical history was included as part of the screening questionnaire. Interestingly 29 of 193 prisoners admitted to having been diagnosed as Human Immuno-deficiency Virus (HIV) positive, some already having progressed to Acquired Immuno-deficiency Syndrome (AIDS) and on highly active anti-retroviral therapy (HAART). This equates to a minimum of $15.0 \%$ recruited in the study who are HIV positive. It is crucial to take cognizance of the fact that this is not an accurate reflection since this was determined by self report and not sero-prevalence. Self report is limited twofold; firstly because not all prisoners have been tested for the virus and secondly because some who know their status may have declined disclosure for various reasons. Recent research done at Westville Correctional Services centre showed an HIV sero-prevalence rate of almost 30\%.13 This high prevalence of HIV in prisons is an area of great concern due to the fact that HIV is readily transmissible in prisons (due to factors such as overcrowding, gangsterism, etc) and because HIV is implicated as an aetiological agent in major mental disorders. ${ }^{14}$

\section{Comparison to International studies}

When comparing prevalence rates from this study to those of international studies large variations in rates are discovered. This is due to many factors namely use of differing diagnostic instruments, differing classification systems, differing samples, differing scopes of illness and different periods of interest.

\section{Comparison to Western prisoner populations}

Limited comparisons have been made with western studies as their findings are unlikely to reflect the unique challenges that face low income countries. A systematic review of 23000 prisoners in Western countries by Fazel and Danesh in 2002 revealed the following: 4\% psychotic illnesses, 10\% major depression, 47\% had antisocial personality disorders in men with women showing similar prevalence rates except for decreased rate of antisocial personality disorder among females. ${ }^{15}$

\section{Comparison to non-Western studies}

A recent relevant study conducted in Iran, by Assadi et al, was selected for comparative analysis since it shares South Africa's low income status and challenges. ${ }^{16}$ The major difference between the two studies was the fact that the Iranian study included only sentenced prisoners whilst this study included sentenced and awaiting trial prisoners. The Iranian study found that 57, 2\% of prisoners had a current Axis 1 disorder. $3,1 \%$ of prisoners had a current psychotic disorder; 29, 1\% had a current major depressive disorder and none were diagnosed with bipolar mood disorder. The principal substance of abuse was opioids. Current opioid abuse and dependence were diagnosed in 9, 5\% of participants. Other substances currently misused included cannabis $(0,8 \%)$ and sedative, hypnotic, anxiolytic drugs $(0$, $7 \%$ ). The lifetime prevalence for opioid dependence was 72 , $7 \%$ which, according to the authors, is probably a more accurate reflection of the magnitude of the problem. Lower rates were found for PTSD (0, 7\%) but higher rates for generalized anxiety disorder (5, 7\%) were found as compared to this study.

10, 5\% of prisoners with current Axis 1 disorders were receiving treatment while the majority did not receive any psychiatric intervention, similar findings to our study.

\section{Current mental health services available at Westville Correctional Centre}

In terms of the current existing mental health resources at Westville prison (as at January 2009), the following were available. Two part- time psychiatrists, three full-time psychologists and thirty one social workers. These figures clearly illustrate the gross discrepancy between mental health care needs and services available.

The prison has a hospital to which acutely mentally ill prisoners are admitted if necessary. They are usually put in single rooms away from other non-psychiatric prisoners if they are a danger to themselves or others. There are however no psychiatrically trained nursing staff to manage these prisoners at present. If these prisoners become unmanageable they are then transferred to state psychiatric hospitals.

\section{Benefits of this study}

Being the first ever study measuring the psychiatric morbidity in a South African prison population, this study has successfully provided an evidence base from which further studies can be done and compared, not just at this prison but from other prisons around South Africa. It has demonstrated a high prevalence of serious psychiatric disorders amongst prisoners and subsequent decision-making regarding mental health initiatives can be derived from this data.

\section{Limitations}

The major limitation of the study was the fact that it was not a multi-centre study. A multi-site study would provide a greater wealth of information of prevalence rates of mental disorders amongst the different prisoner populations in South Africa and would enable comparative analyses between them.

Other limitations include the fact that the MINI, although it screens for the major axis 1 disorders, it is not comprehensive as it does not include diagnoses eg dysthymia, somatoform, impulse control disorders, adjustment disorders, etc. The MINI 
also only screens for antisocial personality disorder and not the other personality disorders. It also does not screen for mental retardation.

Another major limitation is the fact that prisoners' reports of past or present psychiatric diagnoses were not confirmed by prison records.

Only 8 females were chosen for this study due to proportional demographic representation, however future studies should increase the number of female participants so as to increase the likelihood of any statistically significant associations.

\section{Conclusion}

The high prevalence of mental disorders found in this study is in keeping with the significant high rates internationally. Of great concern is the large number of prisoners who have mental disorders but remain undetected in the system. These findings present a cogent argument for greater mental health awareness and services at correctional centres. Specific attention should be focused on screening procedures and staff training programmes to detect mental disorders as this will facilitate appropriate identification and intervention, whether psychological or pharmacological. It is highly recommended that a brief screening instrument be completed on prisoners possibly at reception to prison. Many prisoners with mental disorders do not necessarily require urgent psychiatric attention but they do need some form of assistance by health care services.

It is the recommendation of this study that further research be undertaken at correctional centres around the country to determine the prevalence rates of mental disorders amongst prisoners so that findings may be compared and generalized to the whole of South Africa. Future research is also needed to elucidate to what extent the high psychiatric morbidity are causes, consequences or both of imprisonment. Further, research detailing the mental health care services currently available for prisoners should be undertaken and any disparity between mental health care needs and services should also be investigated.

\section{Acknowledgements}

We would like to acknowledge the Department of Correctional Services, Republic of South Africa for giving permission for this study. We gratefully acknowledge Ms Tonya Esterhuizen, Biostatistician, for statistical planning and data analysis. This research was funded by the Hendrik Vrouwes Scholarship Fund c/o BOE Bank, South Africa. We are indeed grateful for their support without which this research would not have been conducted.

\section{References}

1. The Star Newspaper. SA prison population among the world's highest. April 26, 2008. pg 4.

2. Brinded PMJ, Simpson AIF, Laidlaw TM, Fairley N, Malcolm F. Prevalence of psychiatric disorders in New Zealand prisons: a national study. Australia and New Zealand Journal of Psychiatry 2001; 35: 166-173.

3. Butler T, Allnut S, Cain D, Owens D, Muller C. Mental disorder in the New South Wales prisoner population. Australian and New Zealand Journal of Psychiatry 2005; 39: 407-413.

4. Bland RC, Newman SC, Thompson AH, Dyck RJ. Psychiatric disorders in the population and in Prisoners. International Journal of Law and Psychiatry 1998; 21 (3): 273-279.

5. Butler T, Andrews G, Allnut S, Sakashita C, Smith NE, Basson J. Mental disorders in Australian prisoners: comparison with a community sample. Australia and New Zealand Journal of Psychiatry 2006; 40: 272-276.

6. The White Paper on Corrections in South Africa. Department of Correctional Services. Pretoria. 2009.

7. Schwenzer KJ. Protecting Vulnerable Subjects in Clinical Research: Children, Pregnant Women, Prisoners and Employees. Respiratory Care 2008; 53(10):1342-1349.

8. Sheehan DV, Lecrubier Y, Sheehan KH, et al. The Mini International Neuro-psychiatric Interview (MINI): The development and validation of a structured diagnostic psychiatric interview for DSM IV and ICD 10. Journal of Clinical Psychiatry 1998; 59: supplement 20: 22-33.

9. Statistical Package for the Social Sciences. Version 15.0. Chicago, Illinois, USA. 1989-2006.

10. Leggett T, Louw A, Parry C, Pludderman A, Burton P. Drugs and crime in South Africa - A study in three cities. ISS Monograph Series No 69. Cape Town: MRC. 2002.

11. Altermann AI, Rutherford MJ, Cacciola JS, et al. Prediction of seven month methadone maintenance treatment response by four measures of antisociality. Drug and Alcohol dependence. 1998; 49: 217-223.

12. Flisher AJ, Lang $H$, Laubscher $R$, Lombard CF. Suicide trends in SA. Scandanavian Journal of Public Health. 2004; 32(6): 41 1-418.

13. Gow J, Smith G, Goyer K, Colvin M. The socio-economic characteristics of HIV in a South African prison. International Conference on AIDS. Thailand. 2004 July 11-16.

14. Yudofsky SC, Hales RE. Neuropsychiatric and Behavioral Neurosciences. Washington, DC. American Psychiatric Publishing Inc. 2008:765-786.

15. Fazel S, Danesh J. Serious mental disorders in 23000 prisoners: a systematic review of 62 surveys. Lancet 2002; 359:545-550.

16. Assadi SM, Noorzian M, Pakravannejad M, Yahyazadeh O, Aghayan S, Shariat SV, Fazel S. Psychiatric morbidity among sentenced prisoners: prevalence study in Iran. British Journal of Psychiatry 2006; 188:159-164. 